\title{
Influence of saturable absorbers on fiber ring laser sensors
}

\author{
L. Rodriguez-Cobo ${ }^{1,2 *}$, M.A. Quintela ${ }^{1,2}$, R. Ruiz-Lombera ${ }^{1}$, R.A. Perez-Herrera ${ }^{3}$, \\ M. Lopez-Amo ${ }^{3}$ and J.M. Lopez-Higuera ${ }^{1,2}$ \\ ${ }^{1}$ Photonics Engineering Group, University of Cantabria, 39005, Santander (Spain) \\ ${ }^{2}$ CIBER-bbn, Instituto de Salud Carlos III, 28029, Madrid (Spain) \\ ${ }^{3}$ Universidad Publica de Navarra, Dept. of Electrical and Electronic Engineering and Institute of \\ Smart Cities (ISC), Campus Arrosadia S/N, E-31006, Pamplona, (Spain)
}

\begin{abstract}
In this work, a fiber ring laser sensor has been employed to analyze the influence of passive Er-doped fiber acting as saturable absorber. Lasing modes of fiber ring lasers can be reduced by properly locating a saturable absorber that narrows the spectral width of the FBG that selects the lasing wavelength. Employing commercial Er-doped fiber, different configurations have been evaluated, reaching the SLM regime on a ring cavity of several meters. Thus, the achieved strain response of the whole sensor behaves at its mirror FBG, exhibiting linear response to strain.
\end{abstract}

Keywords: Fiber Bragg grating (FBG), erbium doped fiber, saturable absorber, fiber laser, strain sensor

\section{INTRODUCTION}

Erbium doped fiber lasers (EDFL) where fiber Bragg gratings (FBG) are used as oscillation wavelength selectors present an interesting alternative as active optical sensors compared with the sensor systems based on passive FBGs. These exhibit excellent signal level, high optical signal to noise ratio (OSNR) and a narrow linewidth so they are suitable for their application in long-distance sensor systems. However, a number of drawbacks present in these lasers must be overcome before their widespread application. EDFL inevitably present oscillation in multiple longitudinal modes around its central wavelength because they usually have a large cavity length. Furthermore, it has to be noted, that erbium-doped fiber (EDF) has a dominant homogeneous gain broadening behavior at room temperature, which means that the gain provided by the Erbium at a specific wavelength will determine the gain at other wavelengths. This implies that there is a strong mode competition and usually suffers from unstable lasing. When applying EDFL to interrogate optical sensors, this inherent behavior of the EDFL is the major drawback for practical applications. Different techniques have been proposed to try to mitigate these drawbacks; being the most direct one to achieve a single longitudinal mode (SLM) behavior. Typically, these techniques increase both system complexity and cost that makes them unsuitable for many scenarios. For instance, the use of cavities with multiple-rings requires adjusting the length of the cavity and lasing parameters [1]. In self-injection feedback lasers, the SLM behavior is only guaranteed if there are two channels and the power level is similar in both [2]. Other technique consists of adding an ultra-narrow band width phase-shifted fiber Bragg grating (FBG) into the laser cavity, but the use of two combined gratings in the cavity introduces complexity when using this laser as a sensor [3].

In this work, a stable SLM fiber ring laser for sensing purposes with a very simple configuration is presented and experimentally evaluated. In this structure, a piece of unpumped EDF that acts as saturable absorber has been introduced to achieve single-mode behavior [4]. Saturable absorbers have been successfully employed for narrowing different laser configurations [5-7]. In this work, the influence of the saturable absorber position within the cavity laser in the spectral characteristics of the laser output has been analyzed. When the saturable absorber is placed in the optimum position, the SLM operation is experimentally demonstrated in all working conditions, achieving a very high power stability and a good OSNR. This fiber laser has been characterized as a strain sensor system.

\section{WORKING PRINCIPLE}

In a simple way, when the same signal is forced to travel in both directions within an unpumped EDF, they create a standing wave, being the spatial period of light intensity distribution $\Lambda=\lambda / 2 n_{\text {eff }}$ [4], being $\lambda$ the central wavelength of the counter-propagating signal. This intensity modulation affects the absorption coefficient of the unpumped EDF (spatial-hole-burning), exhibiting also a modulation of this period $\left(\Lambda=\lambda / 2 n_{e f f}\right)$ which also provokes a periodical variation of refraction index [4]. Assuming the same power is available in both ways, a small periodical variation of refraction index is present in the umpumped EDF, which can be understood as a very long weak FBG whose bandwidth is much smaller than the original FBG since $L_{E D F} \gg L_{F B G}$.

*luis.rodriguez@unican.es; phone +34 942200877; fax +34 942200877

25th International Conference on Optical Fiber Sensors, edited by Youngjoo Chung, Wei Jin,

Byoungho Lee, John Canning, Kentaro Nakamura, Libo Yuan, Proc. of SPIE Vol. 10323

$103232 \mathrm{H} \cdot$ (c) 2017 SPIE · CCC code: 0277-786X/17/\$18 - doi: 10.1117/12.2263409 


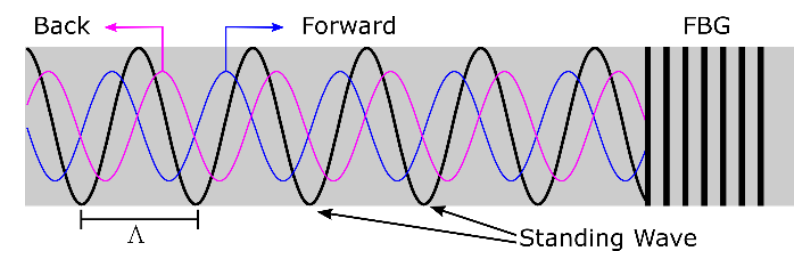

Figure 1. Schematic description of FBG induced by Spatial Hole Burning (SHB) with stationary wave on Er-doped fibers.

Depending on several parameters such as dopant concentration of EDF and power launched to the saturable absorber, the filtering effect described in Fig. 1 might render useless. A trade-off between these parameters is required to obtain a narrowing effect in where the effective length of the induced FBG is much longer than the mirror FBG. Particularly, in this work, a high dopant commercial fiber has been employed to analyze this phenomenon for fiber laser sensors.

\section{EXPERIMENTS}

A proof-of-concept fiber ring laser setup has been employed to analyze the performance of this configuration as a sensor when a saturable absorber is included into the cavity. A commercial Er-doped fiber (4 m of I25 of Fibercore) was employed as active medium within the cavity while it was pumped with a $980 \mathrm{~nm}$ laser. A uniform FBG centered on $1551 \mathrm{~nm}$ with a circulator was used as feedback element to launch the desired wavelengths into the ring cavity. The resulting typical fiber ring laser setup is depicted in Fig. 2. This configuration is modified by introducing a passive EDF block ( $2 \mathrm{~m}$ of I25) first within the non-pumped area of the ring cavity (Fig.2, A) and by introducing it after the circulator (Fig.2 B).

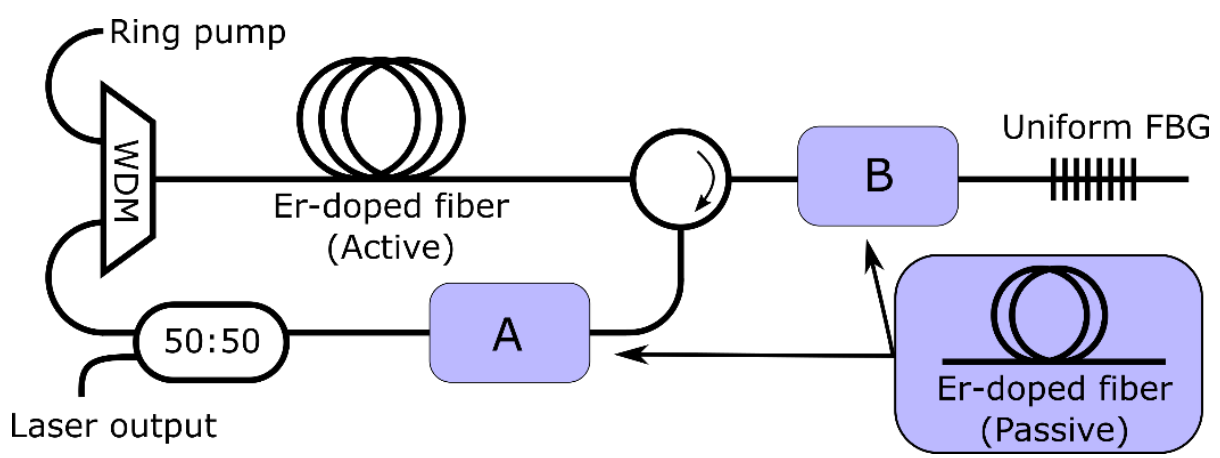

Figure 2. Typical fiber ring laser sensor configuration. A passive Er-doped fiber block is introduced within the ring cavity

(A) and before the FBG (B) to reduce the active lasing modes.

When no passive EDF is employed, the resulting laser works on multimode regime as expected. Same similar happens when the passive fiber is included into the cavity, where laser signal only travels in one direction. The achieved output exhibits less modes, because adding a saturable absorber into the cavity helps to reduce the gain competition on the active fiber. However, when placing the EDF just before the FBG, light travels in both directions achieving the narrowing effect explained in Section 2 and outputting a laser working on SLM regime. A further characterization of these properties has been performed.

\subsection{Spectral characterization}

The first tests have been employed to verify the SLM operation. Employing a high resolution Optical Spectrum Analyzer (BOSA-C of Aragon Photonics) with a resolution of $0.08 \mathrm{pm}$, the three different configurations have been measured while the pump power was maintained at $100 \mathrm{~mW}$ at $980 \mathrm{~nm}$. Besides, the laser output has been mixed with the signal of a Tunable Laser Source (TLS) using a $3 \mathrm{~dB}$ coupler to perform the heterodyne detection. The TLS (Agilent 8164B) has a full-width at half maximum (FWHM) linewidth of $100 \mathrm{kHz}$ and its wavelength has been placed close to the manufactured laser. In Fig.3, both spectra: optic (left) and electric (right) are depicted for each tested configuration. 

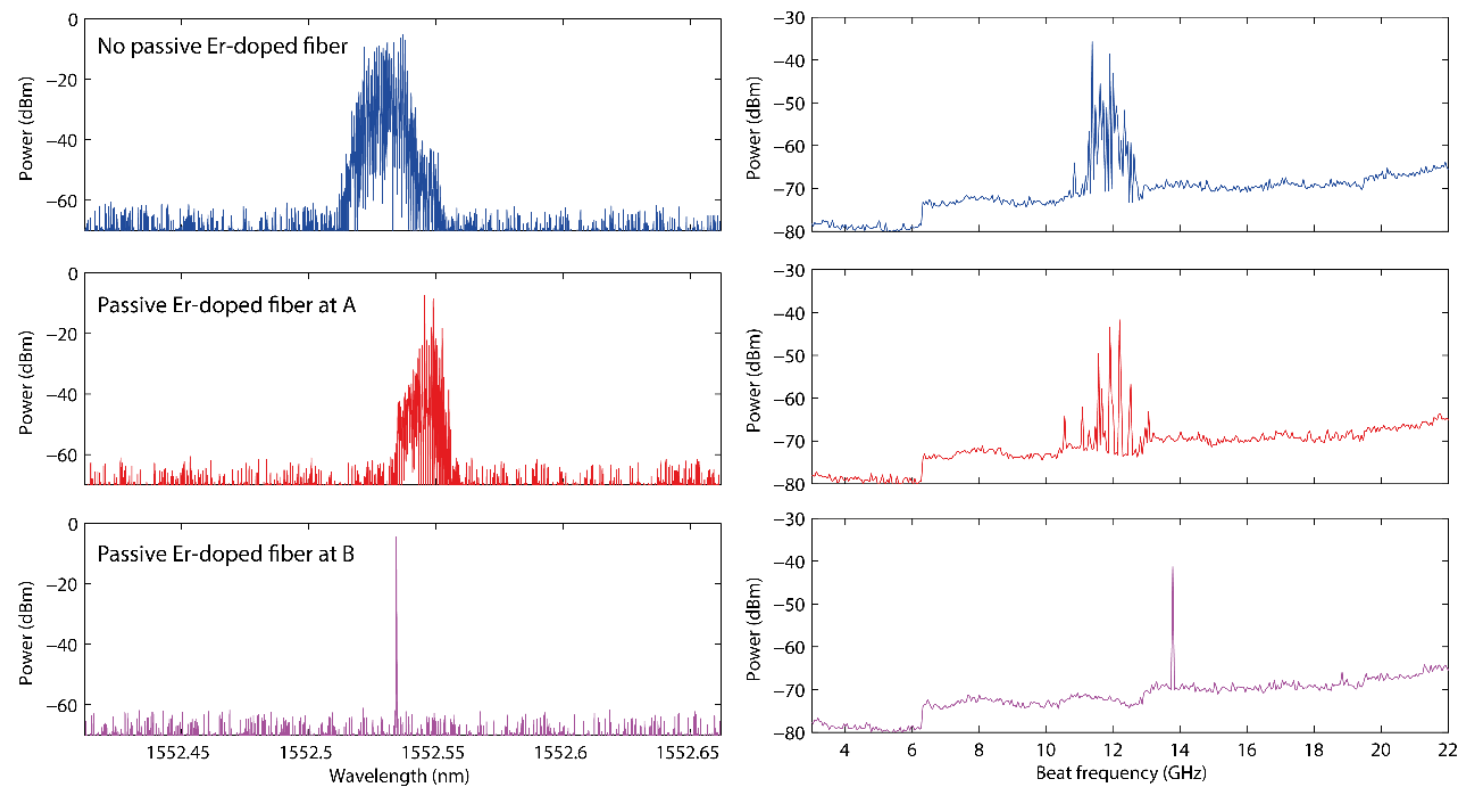

Figure 3. High resolution spectra of each tested configuration (left). Electric beat with a TLS of the laser output for each tested configuration (right). Adding a saturable absorber before the FBG, SLM regime is achieved.

In Fig 3, high resolution spectra and beat frequency of each of the three tested configurations are depicted. When no EDF is included into the laser, the laser outputs several active modes. When a passive EDF is included within the loop, it helps to reduce the simultaneous lasing wavelengths at the active EDF; however, it still maintains several modes. On the contrary, when the EDF is placed before the FBG, the resulting laser exhibits a very stable SLM operation due to the narrowing effect provoked by the saturable absorber (passive EDF).

\subsection{Power stability}

Maintaining the pump constant $(100 \mathrm{~mW}$ at $980 \mathrm{~nm})$, the output power of each configuration was measured. The output laser signal was directly connected to an OSA (HP70952B) to study the power behavior of the proposed setup. The achieved power for each configuration was $+5.3 \mathrm{dBm}$ (no passive EDF), $-1 \mathrm{dBm}$ (passive EDF at A) and $-3.9 \mathrm{dBm}$ (passive EDF at B). As expected, there was a power reduction when a saturable absorber was included into the cavity; however, the power values for the SLM configuration were enough for its application as a sensor. Maintaining the pump constant $(100 \mathrm{~mW}$ at $980 \mathrm{~nm})$, the generated output optical power with passive Er-doped fiber before the FBG (A) was monitored for more than 60 min using the OSA. The output power stability measured each 30 seconds (Fig. 3, left) is depicted, achieving a drift of $0.12 \mathrm{~dB}$ with a $90 \%$ confidence level. For the pump power employed during the stability test, the output power laser exhibited an OSNR higher than $40 \mathrm{~dB}$ (Fig. 3, right).
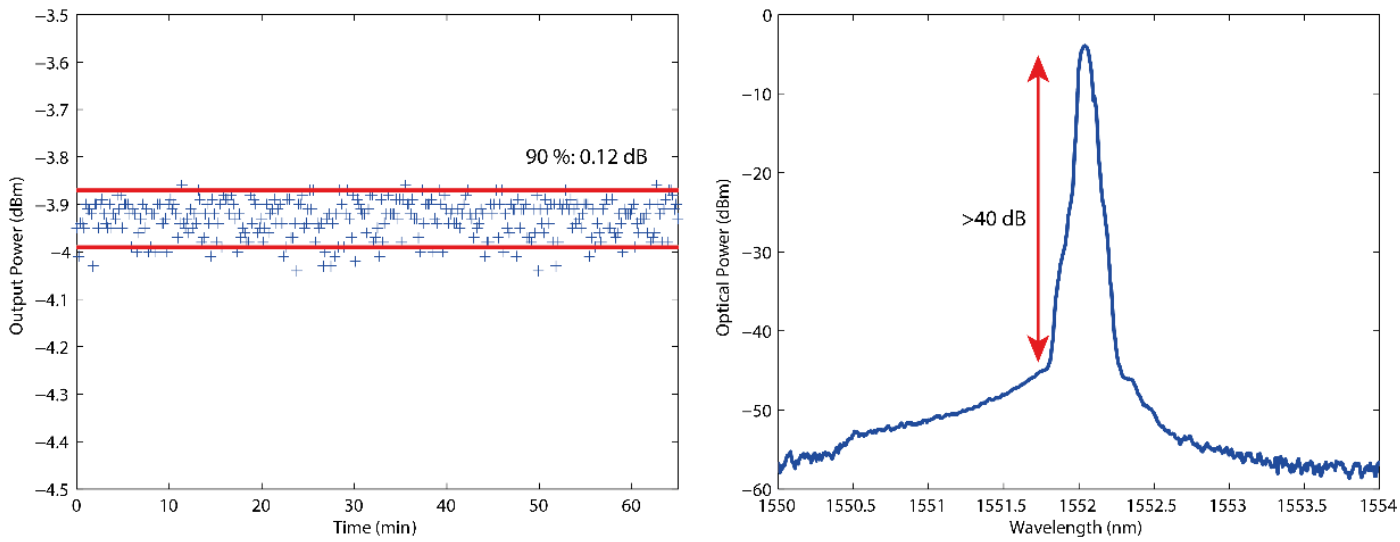

Figure 4. Power stability (left) measured for $60 \mathrm{~min}$ with constant pump power $(100 \mathrm{~mW})$. Output spectrum (right). 


\subsection{Strain response}

After the spectral and power characterization of each configuration, the achieved SLM fiber laser has been tested for strain measurements. Maintaining the EDF block just before the sensing FBG, the laser output has been connected to both OSA and BOSA. The mirror FBG of the laser has been attached to a micrometric linear motor stage to perform a strain sweep reaching a peak deformation above $1500 \mu \epsilon$ while its temperature remained constant $\left(25^{\circ} \mathrm{C}\right)$. The achieved results are depicted in Fig. 5. As expected, the response of the fiber laser sensor structure to strain variations exhibits the same linear response to strain as its reflecting FBG while the SLM regime is maintained all over the tested range.
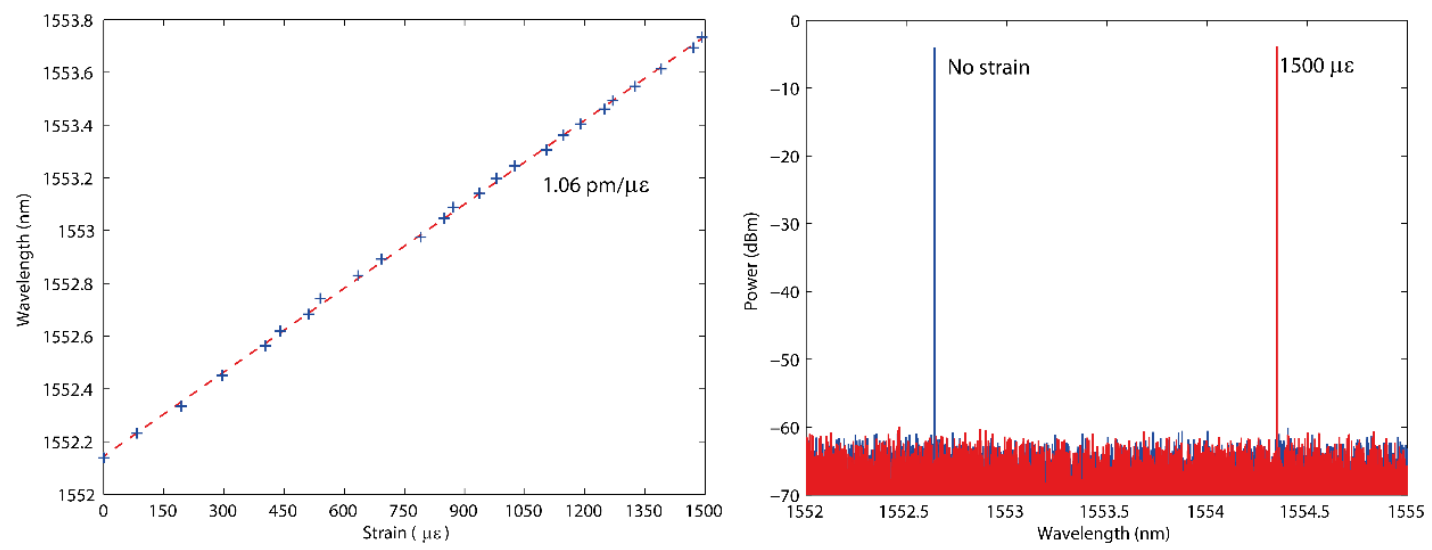

Figure 5. Strain sensitivity of the tested fiber laser sensor (left). BOSA captures to analyze the SLM regime with strain (right).

\section{CONCLUSIONS}

In this work, the influence of introducing a passive EDF into a ring laser configuration acting as saturable absorber has been analyzed. Starting with a typical fiber ring laser that employs a FBG to select the lasing wavelength, an EDF block has been introduced within the passive area of the ring cavity and just before the mirror FBG. As expected, the lasing modes are reduced by adding a saturable absorber into the cavity. However, this effect is especially relevant when the saturable absorber is placed just before the FBG, creating a virtual FBG much narrower than the original seed, forcing the laser to operate into SLM regime. This scheme has been evaluated for sensing purposes achieving the same linearity as expected of FBGs. This work has been supported by the project TEC2013-47264-C2 and TEC2016-76021-C2, of the Spanish government and by a Parliament of Cantabria postdoc grant.

\section{REFERENCES}

[1] S. Pan, X. Zhao, and C. Lou, "Switchable single-longitudinal-mode dual-wavelength erbium-doped fiber ring laser incorporating a semiconductor optical amplifier," Optics letters, 33(8), 764-766 (2008).

[2] M. A. Quintela, R. A. Perez-Herrera, I. Canales et al., "Stabilization of dual-wavelength erbium-doped fiber ring lasers by single-mode operation," IEEE Photonics Technology Letters, 22(6), 368-370 (2010).

[3] S. Rota-Rodrigo, L. Rodríguez-Cobo, M. Á. Quintela et al., "Dual-wavelength single-longitudinal mode fiber laser using phase-shift Bragg gratings," IEEE Journal of Selected Topics in Quantum Electronics, 20(5), 161165 (2014).

[4] K. Zhang, and J. U. Kang, "C-band wavelength-swept single-longitudinal-mode erbium-doped fiber ring laser," Optics express, 16(18), 14173-14179 (2008).

[5] S. Frisken, "Transient Bragg reflection gratings in erbium-doped fiber amplifiers," Optics letters, 17(24), 17761778 (1992).

[6] Y. Song, S. Havstad, D. Starodubov et al., "40-nm-wide tunable fiber ring laser with single-mode operation using a highly stretchable FBG," IEEE Photonics Technology Letters, 13(11), 1167-1169 (2001).

[7] J. Liu, J. Yao, J. Yao et al., "Single longitudinal mode multi-wavelength fiber ring lasers." Optical Fiber Communication Conference, Los Angeles, ThB2, 1-55752-767-9 (2004) 